\title{
FOREGROUND GALAXIES AND THE VARIABILITY OF LUMINOUS QUASARS
}

\author{
J. VON LINDE, U. BORGEEST, J. SCHRAMM AND S. REFSDAL \\ Hamburger Sternwarte \\ Gojenbergsweg 112, D-21029 Hamburg, Germany \\ email: jlinde@hs.uni-hamburg.de \\ AND \\ E. VAN DROM \\ Université de Liège, Inst. d'Astrophysique \\ 5, Avenue de Cointe, B-4000 Liège, Belgium
}

In order to look for an amplification bias (AB) by gravitational lensing caused by medium redshift $(0.2 \lesssim \mathrm{z} \lesssim 0.8)$ clusters or groups of galaxies, we compare galaxy counts in deep CCD images of highly luminous, high redshift QSOs with those in nearby control fields at a distance of $1 \mathrm{deg}$ at the same galactic latitude. The total sample contains 37 objects up to now, from which one field had to be excluded because of a seeing difference between the QSO and control fields.

Observations were done at the DSAZ 3.5 and $2.2 \mathrm{~m}$ telescopes at Calar Alto, Spain, the ESO NTT, Chile, and the Nordic Optical Telescope (NOT) at La Palma. Quasars were selected with respect to optical luminosity (12 QSOs, $\mathrm{z} \geq 1 \mathrm{M}_{V} \leq-29.0 ; \mathrm{H}_{0}=50 \mathrm{~km} \mathrm{~s}^{-1} \mathrm{Mpc}^{-1}, \mathrm{q}_{0}=0$ ), and to both optical luminosity and radio flux (25 QSOs, $\mathrm{z} \geq 1.5, \mathrm{M}_{V} \leq-27.0 \mathrm{mag}, \mathrm{S}(6 \mathrm{~cm})$ $\geq 0.8 \mathrm{Jy}$ ). All fields were observed in the Johnson $\mathrm{R}$ band. Object search and classification was done by an automatic procedure.

Galaxy counts have been analyzed 1 . in the entire fields, 2. inside a circle with radius 1 arcmin around the quasars and the quasar position in the control fields, respectively, and 3. inside a circle with radius 15 arcsec around the quasars, one star in the quasar field, and two stars at the same positions in the control fields (as far as possible), respectively. Since the automatic search is not complete in the direct vicinity of the quasars/stars, this was done by eye. The 4 fields observed at the NOT have been excluded from 1 . and 2 . because of the small field. 
The table shows the excess factors $\mathrm{N}_{q s o} / \mathrm{N}_{\text {contr }}$ and the number of fields $\mathrm{N}_{f}$ used for the different scales for the entire sample and the 2 subsamples of extremely radio loud $\left(\mathrm{S}_{6} \geq 1 \mathrm{Jy}\right)$ and radio quiet QSOs, respectively. One sigma errors based on Poisson statistics are given.

\begin{tabular}{|l|c|c|c|}
\hline & \multicolumn{3}{|c|}{$q=N_{q s o} / N_{\text {contr }}$} \\
\hline Sample & $4^{\prime \prime} \leq \mathrm{r} \leq 15^{\prime \prime 1}\left(\mathrm{~N}_{f}\right)$ & $4^{\prime \prime} \leq \mathrm{r} \leq 60^{\prime \prime}\left(\mathrm{N}_{f}\right)$ & entire fields $^{2}\left(\mathrm{~N}_{f}\right)$ \\
\hline all & $1.61 \pm 0.44(36)$ & $1.15 \pm 0.10(32)$ & $1.035 \pm 0.033(32)$ \\
S $>1 \mathrm{Jy}$ & $1.38 \pm 0.43(23)$ & $1.00 \pm 0.11(19)$ & $1.03 \pm 0.04(19)$ \\
opt & $3.43 \pm 2.25(7)$ & $1.55 \pm 0.30(7)$ & $1.38 \pm 0.14(7)$ \\
\hline
\end{tabular}

${ }^{1}$ Background counts are averaged over the 3 stars

${ }^{2}$ Mind that the fields differ in size

We confirm the excess of galaxies around QSOs on small scales reported by other authors (cf. Van Drom et al.1993) which decreases towards larger radii. The strongest effect is found in the subsample of radio quiet objects, in contradiction to the multiple wave band bias (Borgeest et al. 1991). The reason for this (if not due to statistical fluctuations) could be the higher average absolute brightness of this sample or selection effects in the optical quasar surveys.

The results cannot be interpreted as evidence for an $\mathrm{AB}$ due to clusters of galaxies. However, several faint clusters can be seen in the exposures. Due to the spatial correlation of galaxy clusters, the distance of the control fields might be too small to detect a significant excess in the QSO fields on larger scales.

A very preliminary analyses of light curves of 22 of the objects from the Hamburg Quasar Monitoring (HQM) project (Schramm et al.1994 and references therein) gives no evidence for a correlation of distance and magnitude of the foreground galaxies and the variability of the QSOs. The light curves show at least no strong indication for an $\mathrm{AB}$ caused by microlensing of the quasars by stars in the foreground galaxies.

The complete poster including light curves and contour plots can be obtained from the authors.

\section{References}

Borgeest U., von Linde J., \& Refsdal, S., 1991, A\&A, 251, L35

Schramm, K.-J., Borgeest, U., Kühl, D., v. Linde, J., \& Linnert, M.D., 1994, A\&A Suppl, 106,349

Van Drom E., Surdej J., Magain P., Hutsemèkers D., Gosset E., Claeskens J.F., Shaver P., \& Melnick J., 1993 in Gravitational Lenses in the Universe, eds. J. Surdej et al., (Liège: Université de Liège) 301 